\title{
Molecular Diversity of Staphylococcus aureus from the Nares of Hospital Personnel, HIV-Positive and Diabetes Mellitus Patients in Yaounde Cameroon
}

\author{
Agnes Eyoh ${ }^{1,2 *}$, Marthie Ehlers' ${ }^{3}$, Emilia Lyonga Mbamyah', ${ }^{1,2}$, John Antiabong3, \\ Charles Fokunang2, Marleen Kock ${ }^{3}$, Marie Claire Okomo Assoumou1,2, Michel Toukam², \\ Hortense Gonsu Kamga ${ }^{2}$, George Ikomey ${ }^{1,2}$, Martha Mesembe ${ }^{1}$, Mandi Henshaw ${ }^{4}$, \\ Christiana Haddison ${ }^{4}$, Sinata Koulla-Shiro ${ }^{2,4}$
}

${ }^{1}$ Center for the Study and Control of Communicable Diseases, Faculty of Medicine and Biomedical Sciences, The University of Yaounde I, Yaoundé, Cameroon

${ }^{2}$ Faculty of Medicine and Biomedical Sciences, The University of Yaounde I, Yaoundé, Cameroon

${ }^{3}$ Department of Medical Microbiology, University of Pretoria/National Health Laboratory Service (NHLS), Pretoria, South Africa ${ }^{4}$ Ministry of Public Health, Yaoundé, Cameroon

Email: *e_eyoh@yahoo.com

How to cite this paper: Eyoh, A., Ehlers, M., Mbamyah, E.L., Antiabong, J., Fokunang, C., Kock, M., Assoumou, M.C.O., Toukam, M., Kamga, H.G., Ikomey, G., Mesembe, M., Henshaw, M., Haddison, C. and Koulla-Shiro, S. (2021) Molecular Diversity of Staphylococcus aureus from the Nares of Hospital Personnel, HIV-Positive and Diabetes Mellitus Patients in Yaounde Cameroon. Advances in Microbiology, 11, 740-756.

https://doi.org/10.4236/aim.2021.1112053

Received: October 13, 2021

Accepted: December 27, 2021

Published: December 30, 2021

Copyright $\odot 2021$ by author(s) and Scientific Research Publishing Inc. This work is licensed under the Creative Commons Attribution International License (CC BY 4.0).

http://creativecommons.org/licenses/by/4.0/

(c) (i) Open Access

\begin{abstract}
Nasal carriage of Staphylococcus aureus has been identified as a risk factor for the development of staphylococcal infections caused by endogenous colonizing strains. Information on the genotypic diversity of Staphylococcus aureus is relevant for managing epidemiological and clinical challenges resulting from the evolutionary differences of this bacterium. The objective of this study was to determine and compare the molecular diversity of Staphylococcus aureus isolates from three high-risk populations in Yaounde, Cameroon. Molecular analysis confirmed that $95 \%$ of 100 tested isolates were $S$. aureus. The mecA and Panton Valentine-Leukocidin (PVL) genes (lukS/F-PV) were detected in 37\% (35/95) and 43\% (41/95) of isolates respectively and $18 \%$ $(17 / 95)$ of the isolates harboured both the mecA and lukS/F-PV genes. A mixed distribution of both methicillin sensitive $S$. aureus (MSSA)/PVL and methicillin resistant $S$. aureus (MRSA)/PVL strains were detected within the study population. Community associated MRSA accounted for 94\% (33/35) of the isolates, further classified into allotypes SCCmec type IV 54\% (19/35) and SCCmec type V 40\% (14/35), while two isolates were hospital associated SCCmec type II strains. A majority of the isolates harboured a single aggressive gene regulator allele agr type I. Pulsed Field Gel Electrophoresis (PFGE) generated 18 pulsotypes that grouped isolates irrespective of the study popu-
\end{abstract}


lation. Multilocus Sequence Typing (MLST) of 12 selected isolates was assigned to six pandemic clonal complexes (CC): CC5 (ST5), CC8 [ST8, ( $\mathrm{n}=$ 3)], CC15 (ST 15), CC25 (ST 25), CC72 [ST72 (n = 2)] and CC121 [ST 121 (n =2)] and three atypical sequence types ST 508, ST 699 (CC45) and ST 1289 (CC 88). The study population represents an important reservoir for MRSA, MRSA-PVL and MSSA-PVL which could serve as focal point for further dissemination bringing about significant clinical and epidemiological implications. The predominance of SCCmec IV and agr types in this setting warrants further investigation. Isolates were genetically diverse with MLST indicating that pandemic ST8 was predominant. Detection of atypical STs has provided an insight into the necessity for constant monitoring.

\section{Keywords}

Nasal Carriage, Methicillin Resistant $S$. aureus, Methicillin Sensitive $S$. aureus, Panton-Valentine Leukocidin, Multilocus Sequence Typing

\section{Introduction}

Staphylococcus aureus is a Gram positive bacterium that is part of the microbiota of human nares, skin, intestine, upper respiratory tract and vagina [1]. These bacteria can cause serious disease when there is a breach of the mucosal barrier allowing sterile body sites to be infected by tissue invasion or toxin production [2]. The host and bacterial factors that facilitate colonization have not been fully characterized. However, polymorphism of genes that encode the glucocorticoid receptor, interleukin-4, complement inhibitor proteins and the reduced expression of antimicrobial peptides in nasal secretions have been associated with persistent nasal carriage [3] [4].

Bacteria adhesion is facilitated by loricrin, a major component of the squamous epithelium, which is the primary ligand for the clumping factor B (ClfB) expressed on the surface of $S$. aureus. Other bacterial factors like cell wall teichoic acid, capsular polysaccharide and iron-regulator surface determinants are also involved in Staphylococcus aureus colonization [5] [6] [7] [8]. Invasiveness of the bacteria is enhanced by the secretion of enzymes (nucleases, proteases, lipases, hyaluronidase and collagenases) and cytokines that inhibit complement activation, cause neutrophil chemotaxis and neutralize antimicrobial defensin peptides [9] [10]. Some strains produce toxic proteins such as enterotoxins, exfoliative toxins and leucocidin, which are responsible for diseases that vary in severity from superficial skin lesions to more serious invasive and life-threatening infections such as pneumonia, endocarditis, septicaemia, acute staphylococcal toxemia syndromes and staphylococcal food poisoning [11].

In vulnerable persons, colonization increases the risk of subsequent infection usually caused by colonizing strains [12]. Several studies have illustrated a causal association between $S$. aureus nasal carriage and an estimated 4- to 10-fold increase in the odds of carriers developing staphylococcal disease [13] [14]. The 
Human Immunodeficiency Virus/acquired immune deficiency syndrome (HIV/AIDS) associated infections are generally linked to the progressive depletion of cluster of differentiation $4 \mathrm{~T}(\mathrm{CD} 4 \mathrm{~T})$ cells. A count lower than 200 cells $/ \mathrm{mm}^{3}$ of blood, recent antibiotic use, duration of hospital stay are considered to be risk factors for nasal colonization by methicillin, resistant $S$. aureus in HIV-infected patients [15] [16]. Diabetes mellitus, a debilitating chronic disease with ensuing complications such as renal failure, dermatological disorders and peripheral neuropathy with risk of foot ulcers, is considered risk factors for MRSA colonization [17]. Colonization of hospital personnel has been identified as a reservoir of healthy carriers of $S$. aureus in the hospital and community. Previous studies in Ethiopia, recorded a prevalence of $28.8 \%$ (34/118) S. aureus colonization of hospital personnel, among which $44.1 \%$ (15/34) were resistant to methicillin [18]. A similar study conducted in Yaounde Cameroon, recorded a prevalence of $23.7 \%$ [19]. Transmission of resistant strains from personnel to patients is likely to occur during routine patient care in the absence of effective hand hygiene. Acquisition of MRSA on hands by healthcare workers, was common after examination of sites like the chest, abdomen, forearm and hands [20].

Management of staphylococcal infections has become a burden to the global health community leading to increased cost of healthcare, prolonged hospital stay and higher morbidity and mortality among infected individuals [21] [22]. The epidemiology of $S$. aureus especially MRSA has evolved considerably over the years. Managing the upsurge in prevalence of community associated methicillin resistant Staphylococcus aureus (CA-MRSA) is challenging for several healthcare institutions which are already battling with high levels of hospital-associated methicillin resistant Staphylococcus aureus (HA-MRSA) [23]. Disease severity is further compounded by the presence of mobile genetic elements that confer antibiotic resistance such as Staphylococcus cassette chromosome (SCC) harboring mec elements for methicillin resistance or the $A$ operon for vancomycin resistance. The SCCmec type II strains initially associated to the hospital settings have now been identified among community and animal isolates [24] [25]. Methicillin sensitive $S$. aureus (MSSA) and MRSA strains harboring Panton-Valentine Leukocidin (PVL), a bi-component cytotoxin encoded by luk $S-P V$ with diverse genetic characteristics, are on the rise in Africa [26]. Given that $l u k S-P V$ is phage mediated and can easily be transmitted between isolates, this may explain high prevalence rates of gene among strains in Africa where monitoring is limited. PVL-encoding operon has been detected in 55.9\% of $S$. aureus strains from certain African populations such as the Babongo Pygmies. Data from across Europe, however, depicts lower rates: $1.8 \%$ in Ireland, $4 \%$ in Turkey, 30\% in Germany and 20\% in United Kingdom [27] [28]. The expression of PVL and other $S$. aureus virulence determinants is thought to be controlled by multiple regulatory pathways including the accessory gene regulator (agr) characterized by a polymorphism of its auto-inducing peptide (AIP). Based on this polymorphism, $S$. aureus has been classified into four agr groups. Furthermore, molecular typing techniques such as pulsed field gel electrophore- 
sis (PFGE) and multilocus sequence typing (MLST) are currently being used to generate genotype information to categorize isolates into sequence types (STs) and clonal lineages or complexes (CCs) for effective epidemiological surveillance and for monitoring evolutionary trends [29].

The clinical relevance of characterizing both MRSA and MSSA has been demonstrated though such data is limited in Cameroon. The objective of this study was therefore to investigate the genetic diversity of $S$. aureus isolates obtained from hospital personnel, HIV-positive and diabetes mellitus patients in Yaounde, Cameroon for disease surveillance and better patient management.

\section{Materials and Methods}

\subsection{Sample Collection}

Nasal samples were collected from hospital personnel, HIV-positive patients and diabetes mellitus out-patients in three health institutions over a period of 12 months, from January 2016 to January 2017 Samples were collected from participants by introducing a sterile cotton swab into both nostrils one after the other. Collected nasal samples were transported in an enrichment medium (m-Staphylococcus broth) containing $10 \%$ Sodium Chloride $(\mathrm{NaCl})$ to the bacteriology unit of the Centre for the Study and Control of Communicable Diseases, Faculty of Medicine and Biomedical Sciences of the University of Yaounde 1, for culture and identification. One hundred randomly selected multidrug resistant and biochemically identified Staphylococcus aureus isolates were further analyzed. Isolates were selected according to study population and study site as follows: hospital personnel: [Yaounde General Hospital $(n=6)$, Yaounde Central Hospital $(\mathrm{n}=10)$ and Biyem-Assi District Hospital $(\mathrm{n}=4)]$. HIV-positive patients: [Yaounde Central Hospital $(n=60)$ ] and diabetes mellitus patients: [Yaounde Central Hospital $(\mathrm{n}=20)$ ].

\subsection{Culture and Identification of Staphylococcus aureus Isolates}

Identification was based on mannitol fermentation after incubation of plates at $37^{\circ} \mathrm{C}$ for 24 to 48 hours. Isolates that fermented mannitol were further analysed using API staph (BioMérieux, Marcy l'Etoile, France). Antimicrobial susceptibility testing was carried out according to the disk diffusion method by Kirby Bauer. Identified isolates were stored at $-20^{\circ} \mathrm{C}$ pending molecular analyses performed at the Department of Medical Microbiology, University of Pretoria, South Africa.

\subsection{Total Genomic DNA Extraction of Staphylococcus aureus Isolates}

Total bacterial DNA was extracted from a $2 \mathrm{ml}$ tryptone soya broth (Oxoid Ltd., Basingstoke, UK) bacteria suspension. DNA extraction was performed using the Zymo Fungal/Bacterial DNA Mini Prep ${ }^{\mathrm{TM}}$ (Zymo Research Corp CA-USA,) DNA extraction kit according to instructions referenced by the manufacturer 
[20]. Extracted DNA was stored at $-20^{\circ} \mathrm{C}$.

\subsection{Molecular Identification of Staphylococcus, aureus Isolates}

The isolates were subjected to a multiplex Polymerase Chain Reaction (M-PCR) assay using specific primers to confirm biochemical identification. Staphylococcus species and genus specific upstream sense and downstream antisense primers shown in Table 1 were used to amplify the 16SrRNA and the nuc fragments of isolates respectively [30]. The PCR reaction mixture, volume and cycling conditions were adapted as previously described [30] [31]. Generated amplicons were run on $1.5 \%$ agarose gel electrophoresis after staining with $5 \mu \mathrm{l}$ ethidium bromide (10 mg/ml; Sigma Aldrich) and visualized under UV light (Transilluminator Ultra-violet products incorporated, USA). A 100 bp DNA ladder (Promega, Madison, USA) was used as DNA molecular weight standard.

\subsection{Detection of Antibiotic Resistance and Virulence Genes by M-PCR Assays}

Primers specific for mecA were used to amplify the genes responsible for methicillin resistance. The ability to produce Panton-Valentine leukocidin (PVL) was determined by the presence of $l u k S$-PV-lukF-PV and virulence regulation by the different agr (I to IV) groups, according to previously described protocols [28] [32]. Details of primer sequences, expected sizes of the amplified products, reaction volume and specific annealing temperatures are listed in Table 1.

Table 1. The PCR primer sequences (from previous studies) used for molecular confirmation, methicillin resistance, virulence and virulence regulation of $S$. aureus isolates.

\begin{tabular}{|c|c|c|c|c|}
\hline Primer name & ${ }^{*}$ Primer sequence & $\begin{array}{l}\text { Target } \\
\text { gene }\end{array}$ & $\begin{array}{l}\text { Amplicon } \\
\text { size (bp) }\end{array}$ & $\begin{array}{l}\text { Annealing } \\
\text { temp }\left({ }^{\circ} \mathrm{C}\right)\end{array}$ \\
\hline $\begin{array}{l}\text { Staph756 } \\
\text { Staph } 750\end{array}$ & $\begin{array}{l}\text { F/-5AACTCTGTTATTAGGGAAGAACA-3 } \\
\text { R/-5 CCACСTTCCTCCGGTTTG TCACC-3 }\end{array}$ & $16 \mathrm{~S} r R N A$ & 756 & \\
\hline $\begin{array}{l}\operatorname{Tn} 1(+) \\
\operatorname{Tn} 2(-)\end{array}$ & $\begin{array}{l}\text { F/-5GACTATTATTGGTTGATCCACCTG-3 } \\
\text { R/-5GCCTTGACGAACTAAAGCTTCG-3 }\end{array}$ & nuc & 218 & \\
\hline $\begin{array}{l}\operatorname{mec} A 1 \\
\operatorname{Mec} A 2\end{array}$ & $\begin{array}{l}\text { F/-5 AATATCGATGGTAAAGGTTGGC -3 } \\
\text { R/-5 AGTTCTGCAGTACCGGATTTGC-3 }\end{array}$ & mecA & 310 & 57 \\
\hline $\begin{array}{l}\text { lukS-PV } \\
\text { LukF-PV }\end{array}$ & $\begin{array}{l}\text { F/-5 ATCATTAGGTAAAATGTCTGGACATGATCCA-3 } \\
\text { R/-5 GCATCAAGTGTATTGGATAGCAAAAGC-3 }\end{array}$ & $L u k S / F$ & 151 & 57 \\
\hline$a g r I$ & $\begin{array}{c}\text { F/-5 ATGCACATGGTGCACATGG-3 } \\
\text { R/-5 R-GTCACAAGTACTATAAGCTGCGAT-3 }\end{array}$ & $\operatorname{agr} \mathrm{I}$ & 440 & \\
\hline agrII & $\begin{array}{c}\text { F/-5 ATGCACATGGTGCACATGG-3 } \\
\text { R/-5 GTCACAAGTACTATAAGCTGCGAT-3 }\end{array}$ & $\operatorname{agr} \mathrm{II}$ & 572 & 5 \\
\hline agr III & $\begin{array}{c}\text { F/-5 ATGCACATGGTGCACATGG-3 } \\
\text { R/-5 CTGTTGAAAAAGTCAACTAAAAGCTC-3 }\end{array}$ & agrIII & 4064 & 35 \\
\hline $\operatorname{agrIV}$ & $\begin{array}{l}\text { F/-5 ATGCACATGGTGCACATGG-3 } \\
\text { R/-5 CGATAATGCCGTAATACCCG-3 }\end{array}$ & $\operatorname{agr} \mathrm{IV}$ & 588 & \\
\hline
\end{tabular}

${ }^{\star}$ Legend: F/Forward primer: R/Reverse primer. 


\subsection{Identification of the SCCmec Types of MRSA Isolates}

The diversity of MRSA strains was established by multiplex PCR assays of the chromosomal cassette recombinase ( $c c r$ ) types I to V and the Staphylococcal cassette chromosome mec allotypes (classes A to C). SCCmec types were assigned based on the combination of $c c r$ type and mecA classes detected [33].

\subsection{Pulsed Field Gel Electrophoresis}

Pulsed Field Gel Electrophoresis analysis of 95 molecularly confirmed isolates (MSSA $n=60$ and MRSA $n=35$ ) was performed in accordance with CDC (Centers for Disease Control and Prevention) Pulse Net protocol (https://www.cdc.gov/pulsenet/pathogens/protocol-images.html). The reference strain ATCC 12,600 was used for quality control. Briefly, to $400 \mu \mathrm{l}$, each bacterial suspension (optical density 1.2) of an overnight culture was added $20 \mu \mathrm{l}$ of lysozyme $(20 \mathrm{mg} / \mathrm{ml})$ and incubated at $56^{\circ} \mathrm{C}$ for 20 minutes. To this was added $20 \mu \mathrm{l}$ of thawed proteinase $\mathrm{K}$ and $5 \mu \mathrm{l}$ of lysostaphin $(100 \mu \mathrm{g} / \mathrm{ml}$, Sigma). The bacterial suspension for each isolate was thereafter mixed with $1.2 \%$ (400 $\mu$ l) low melting point agarose gel (Seakem) and casted into plugs. Plugs were prepared by cutting genomic DNA embedded within them with Serratia marcescens (SmaI) (Fermentas Life Sciences, St. Leon-Rot, Germany) restriction enzyme. Electrophoresis of DNA fragments was performed on $1 \%$ Seakem Gold agarose gel in $1 \mathrm{X}$ tris-Boronic Acid-EDTA buffer, using the Rotaphor ${ }^{\circledR}$ (Biometra, Gottingen, Germany) at $13^{\circ} \mathrm{C}$ for 25 hours, with a reorientation angle of $120^{\circ}$ and a linear increase of switch time from 5 to 40 seconds. The gels were stained with ethidium bromide ( $2 \mathrm{mg} / \mathrm{mL}$, Sigma) for 30 minutes, destained in ultra-pure water for 15 minutes, viewed under UV light (Transilluminator, Ultra-violet products incorporated, USA), and resulting images photographed and analyzed.

\subsection{Multilocus Sequence Typing (MLST) of Staphylococcus aureus}

Twelve representative isolates based on pulsotypes generated by PFGE were selected for sequencing by MLST. PCR primers designed by Inqaba Biotech South Africa were used to amplify the following highly conserved seven housekeeping gene (carbamate kinase $(\operatorname{arcC})$, shikimate dehydrogenase (aroE), glycerol kinase $(g l p)$, guanylate kinase $(g m k)$, phosphate acetyltransferase ( $p t a)$, triosephosphate isomerase (tpi), and acetyl coenzyme A acetyltransferase ( $y q i \mathrm{~L})$ as previously described (34)). Each pair of primers amplified an internal fragment of a housekeeping gene of approximately $500 \mathrm{bp}$. Sequence analysis was performed by Inqaba Biotech South Africa. The identified loci and corresponding alleles were then compared with those from the $S$. aureus database.

\subsection{Data Analysis}

Data collected were entered into a spreadsheet and analyzed using Stata (version 13.0 STATA corps, Texas, USA). Proportions were compared using Chi-Square tests. The level of statistical significance was set at a $p$-value $\leq 0.05$. PFGE images 
were analysed with GelCompare II software (Applied maths, Kortrijk, Belgium). The percentage of relatedness was determined by Dice coefficient with $1.5 \%$ band tolerance and $1 \%$ optimization and the unweighted pair group method with arithmetic averages (UPGMA). The coefficient of similarity was set at $\geq 80 \%$ to show clonal relationships. Sequence analysis was done using CLC workbench software and serotypes assigned using the online database $S$. aureus (http://saureus.mlst.net/).

\section{Results}

In total, 95 (95\%) of the 100 isolates were confirmed as $S$. aureus based on $16 \mathrm{~S} r R N A$ and nucA genes (Figure 1). The other five isolates were not subjected to any further analysis. These 95 confirmed $S$. aureus were distributed according to study population was as follows; hospital 20, HIV-positive patients 57 and diabetes mellitus patients 18 .

The mecA gene for MRSA, was detected in 36.8\% (35/95) of the analyzed isolates. The other isolates, (60) were identified as MSSA. Distribution of MRSA according to study population was as follows; hospital personnel $45 \%(9 / 20)$, HIV-positive patients 33\% (19/57) and diabetes mellitus patients 39\% (7/18). For the lukS/F-PV gene, $40 \%$ was detected amongst isolates from hospital personnel, $40 \%$ from HIV-positive patients and 56\% from diabetes mellitus patients resulting in an overall prevalence of $43 \%$ (41/95). The combined prevalence for MRSA/PVL was $49 \%$ (17/35) distributed as follows: hospital personnel $41 \%$ (7/17), HIV-positive patients $35 \%(6 / 17)$ and diabetes mellitus patients $24 \%$ (4/17), while that for MSSA/PVL was 40\% (24/60) with the highest proportion $81 \%(17 / 24)$ detected among isolates from the HIV-positive population, followed by diabetes mellitus 25\% (6/24) and hospital personnel 4\% (1/24). Base on study site, the Yaounde Central Hospital registered the highest prevalence of $86 \%$ and $90 \%$ for both mecA and lukS/F-PV genes respectively.

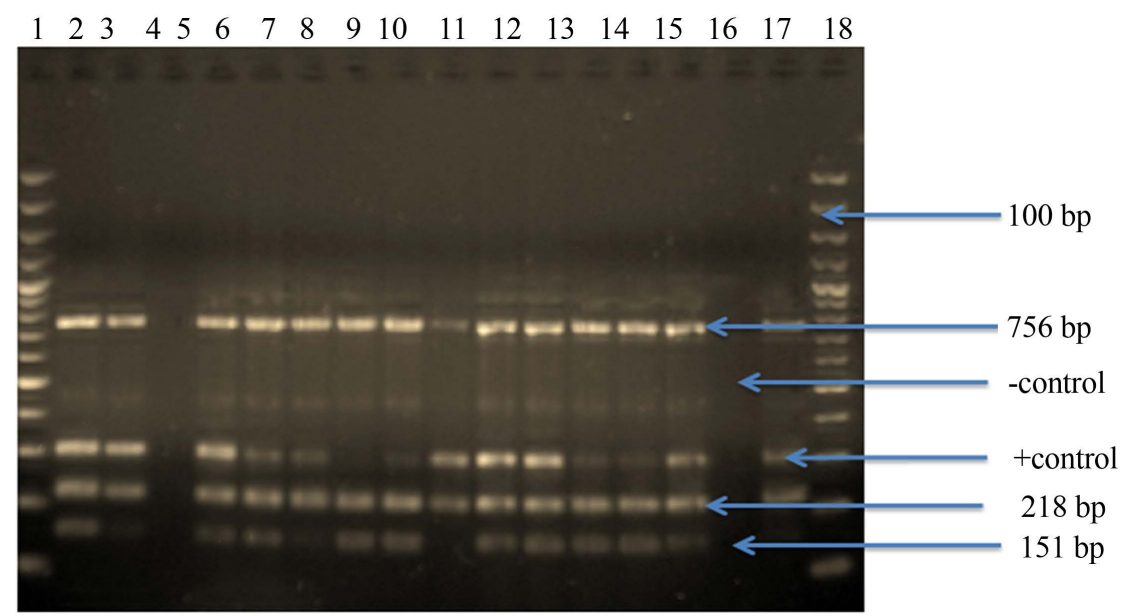

Figure 1. The PCR amplification products of the $S$. aureus encoding 16S rRNA (756 bp), nuc (218 bp), mecA (310 bp) and the lukS/F-PV (151 bp) genes. Lanes 1 and 18:100 bp molecular weight marker. Lanes 2 - 17 S. aureus isolates. 
Overall, 71\% (25/35) of the MRSA were typed as community acquired CA-MRSA SCCmec type IV, SCCmec type V 23\% (8/35) and two isolates (6\%) typed as SCCmec type II (hospital acquired-MRSA). Analyses revealed that 55\% (5/9), 79\% (15/19) and 71\% (5/7) of MRSA from hospital personnel, HIV-positive and diabetes mellitus patients respectively, were typed as SCCmec type IV. All the MRSA/PVL positive isolates $(\mathrm{n}=6)$ from HIV-positive subjects belonged to SCCmec type IV. The two hospital acquired-MRSA SCCmectype II was detected among isolates from the Yaounde Central Hospital. According to study population, the predominant agr type was agr type I distributed as follows; hospital personnel 65\% (13/20), HIV-positive patients 52\% (30/57) and diabetes mellitus patients $33 \%(6 / 18)$. These results and more are shown in Figure 2.

\subsection{Pulsed Field Gel Electrophoresis}

Of the 95 (35 MRSA and 60 MSSA) isolates that underwent restriction digestion with Smal and electrophoresis on agarose gel, 93 had PFGE detected patterns. Thus 2 MSSA isolates were untypable with Sma1. These two isolates were not analysed any further. Relatedness of strains was analyzed by constructing a dendrogram based on distance value and $80 \%$ homology. The dendrogram showed 18 distinct pulsotype designated here as CI to CVIII (Figure 3) and several singletons. Twelve representative isolates based on generated pulsotypes were sequenced using MLST.

\subsection{Molecular Diversity of $S$, aureus Based on MLST}

Six pandemic STs (ST5, ST8, ST15, ST 25, ST 72 and ST 121) and three atypical STs (ST 508, ST 669 and ST 1289) were identified among the 12 isolates analyzed by MLST (Table 2). Results illustrates that the six pandemic STs belonged to six major clonal complexes [CC5 (ST5), CC8 (ST8), CC15 (ST15), CC25 (ST25), CC72 (ST72 and CC121 (ST121)]. The predominant STs were ST8 $(n=3)$ and ST72 $(n=2)$. The sequenced isolates and the characteristics of the identified STs are shown below in Table 3 and Table 4 respectively.

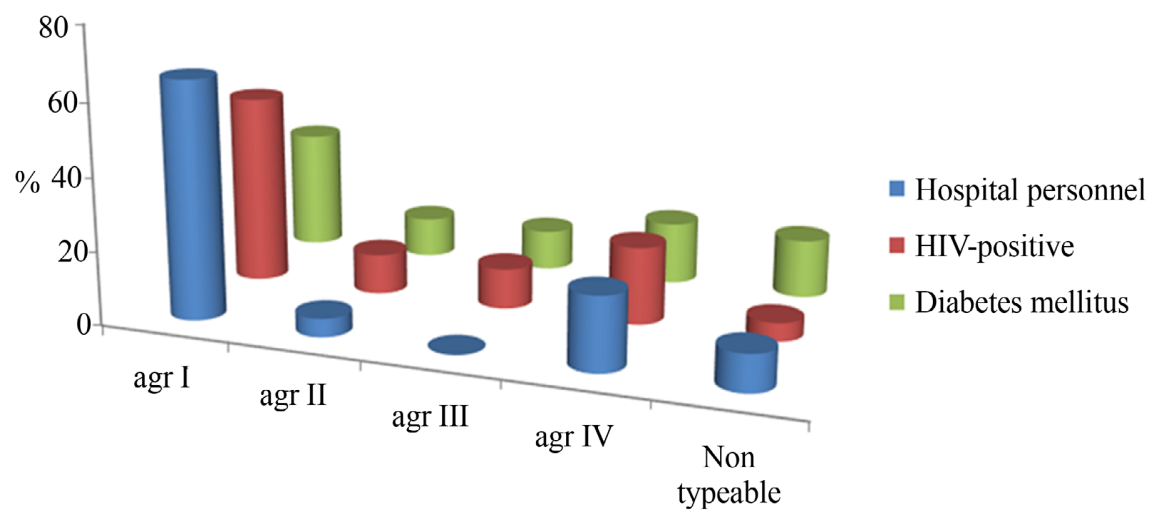

Figure 2. Percentage distribution of agr groups among the $S$. aureus isolates investigated. 
Table 2. Comparison of methicillin -resistant $S$. aureus and PLV harboring carrier state among study groups.

\begin{tabular}{cccc}
\hline $\begin{array}{c}\text { Staphylococcus aureus } \\
\text { isolates }\end{array}$ & $\begin{array}{c}\text { Hospital personnel } \\
(\mathrm{n}=20)\end{array}$ & $\begin{array}{c}\text { HIV-positive Subjects } \\
(\mathrm{n}=57)\end{array}$ & $\begin{array}{c}\text { Diabetes Mellitus patients } \\
(\mathrm{n}=18)\end{array}$ \\
\hline $\begin{array}{c}\text { Methicillin-resistant } \\
\text { Staphylococcus aureus } \mathrm{n}(\%)\end{array}$ & $9(45 \%)$ & $19(33 \%)$ & $7(39 \%)$ \\
$\begin{array}{c}\text { Panton Valentine leucocidin } \\
\text { producing strains n }(\%)\end{array}$ & $8(40 \%)$ & $23(40 \%)$ & $10(56 \%)$ \\
\hline
\end{tabular}

Table 3. Allele numbers and assigned clonal complexes of MLST sequenced $S$. aureus isolates.

\begin{tabular}{|c|c|c|c|c|c|c|c|c|c|c|}
\hline \multirow{2}{*}{ Isolate ID } & \multirow{2}{*}{ Host } & \multirow{2}{*}{$\begin{array}{l}\text { ST based on } \\
\text { MLST-7 }\end{array}$} & \multirow{2}{*}{$\begin{array}{l}\text { Clonal } \\
\text { complex }\end{array}$} & \multicolumn{7}{|c|}{ Loci and corresponding alleles } \\
\hline & & & & $\operatorname{arc}$ & $\operatorname{aroE}$ & $g l p$ & $g m k$ & pta & $t p i$ & yqil \\
\hline BA 07 & Hospital personnel & 72 & 72 & 1 & 4 & 1 & 8 & 4 & 4 & 3 \\
\hline BA 19 & Hospital personnel & 669 & - & 3 & 1 & 94 & 1 & 29 & 5 & 3 \\
\hline HDJ 15 & HIV-positive patient & 15 & 15 & 13 & 13 & 1 & 1 & 12 & 11 & 13 \\
\hline HDJ 16 & HIV-positive patient & 25 & 25 & 4 & 1 & 4 & 1 & 5 & 5 & 4 \\
\hline HDJ 24 & HIV-positive patient & 121 & 121 & 6 & 5 & 6 & 2 & 7 & 14 & 5 \\
\hline HDJ 88 & HIV-positive patients & 8 & 8 & 3 & 3 & 1 & 1 & 4 & 4 & 3 \\
\hline HDJ 127 & HIV-positive patient & 508 & 45 & 10 & 40 & 8 & 6 & 10 & 3 & 2 \\
\hline HDJ 142 & HIV-positive patient & 8 & 8 & 3 & 3 & 1 & 1 & 4 & 4 & 3 \\
\hline HDJ 219 & HIV-positive patient & 8 & 8 & 3 & 3 & 1 & 1 & 4 & 4 & 3 \\
\hline HDJ 213 & HIV-positive patient & 72 & 72 & 1 & 4 & 1 & 8 & 4 & 4 & 3 \\
\hline HJD 229 & HIV-positive patient & 5 & 5 & 1 & 4 & 1 & 4 & 12 & 1 & 10 \\
\hline HDJ 245 & HIV-positive patient & 1289 & 88 & 22 & 1 & 14 & 10 & 12 & 4 & 31 \\
\hline
\end{tabular}

Legend: Sequence Type (ST) Multilocus Sequence Type (MLST).

Table 4. Genetic characteristics of the twelve sequenced isolates.

\begin{tabular}{cccccccccc}
\hline Isolate ID & (STs) & CCs & MLST allelic profile & mecA & $\begin{array}{c}\text { SCCmec } \\
\text { ype }\end{array}$ & LukS/lukF-PV & agr & Study site & Origin of isolates \\
\hline HJD 229 & 5 & 5 & $1-4-1-4-12-1-10$ & - & IV & - & I & YCH & HIV-positive patients \\
HDJ 219 & 8 & 8 & $3-3-1-1-4-4-3$ & - & V & - & I & YCH & HIV-positive patients \\
HDJ 142 & 8 & 8 & $3-3-1-1-4-4-3$ & - & - & - & I & YCH & HIV-positive patients \\
HJD88 & 8 & 8 & $3-3-1-1-4-4-3$ & + & V & + & II & YCH & HIV-positive patients \\
HDJ15 & 15 & 15 & $13-13-1-1-12-11-13$ & - & - & + & III & YCH & HIV-positive patients \\
HDJ 16 & 25 & 25 & $4-1-4-1-5-5-4$ & - & - & - & & YCH & Diabetes Mellitus \\
HDJ213 & 72 & 72 & $1-4-1-8-4-4-3$ & - & - & + & IV & YCH & HIV-positive patients \\
BA07 & 72 & 72 & $1-4-1-8-4-4-3$ & - & - & - & & Biyem Assi & Hospital personnel \\
HDJ 24 & 121 & 121 & $6-5-6-2-7-14-5$ & + & IV & + & I & YCH & Diabetes Mellitus \\
HDJ127 & 508 & 45 & $10-40-8-6-10-3-2$ & - & - & - & I & YCH & HIV-positive patients \\
BA19 & 669 & & $3-1-94-1-29-5-3$ & - & - & - & IV & Biyem Assi & Hospital personnel \\
HDJ245 & 1289 & 88 & $22-1-14-109-12-4-31$ & + & V & + & I & YCH & HIV-positive patients \\
\hline
\end{tabular}

ST: Sequence Type; YCH: Yaoundé Central hospital; CCs: Clonal Complexes. 


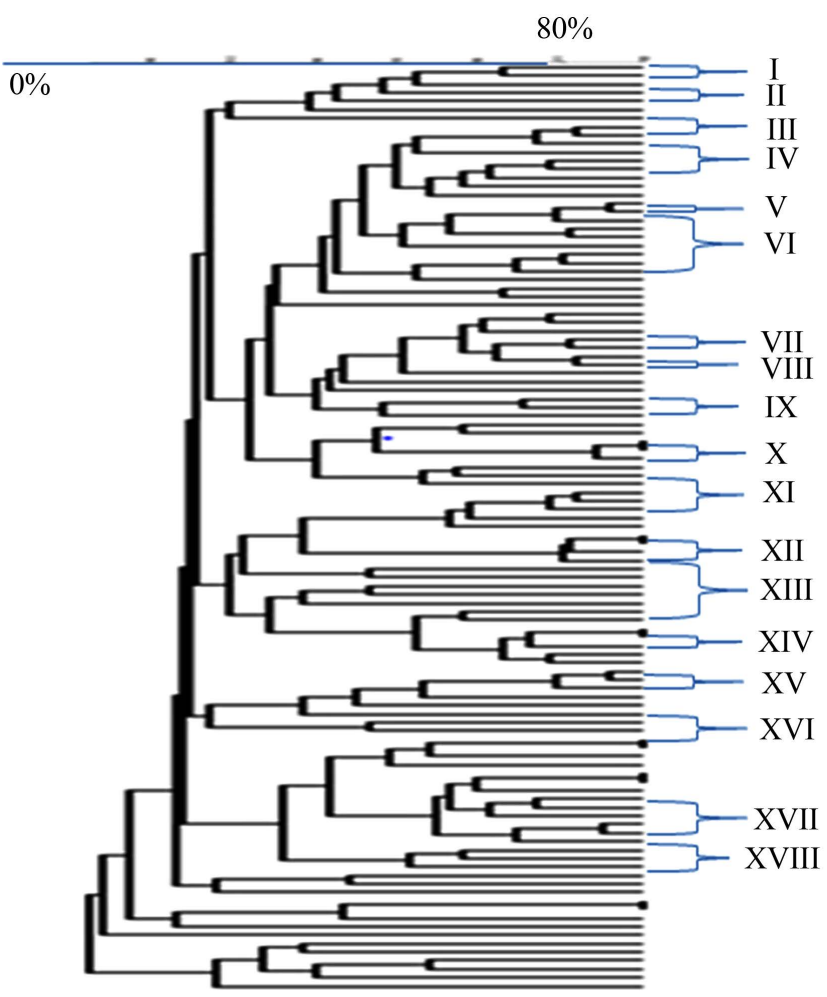

Figure 3. Hierarchical clustering analysis of the pulsed field gel electrophoretic (PFGE) pattern showing the genetic relatedness of the $S$. aureus strains isolated from hospital personnel, HIV-positive and diabetes mellitus patients. The dengrogram was generated by BioNumerics Software with the Band-matching coefficient and the unweighted pair-group method with arithmetic mean (UPGMA).

\section{Discussion}

The present study has demonstrated the importance of molecular identification by revealing that community acquired-MRSA-PVL and community acquired-MSSA-PLV are common amongst $S$. aureus isolates colonizing the nares of hospital personnel, HIV-positive and diabetes mellitus patients in Yaounde. Three atypical sequence types were detected while pandemic strains were found to cluster irrespectively of studied genetic characteristics analysed or study population. Molecular identification and sequence typing of bacterial isolates is essential to confirm speciation, to monitor evolutionary trends and to determine clonal relatedness within the study population.

The prevalence of colonization with MRSA has been reported to vary according to the population studied as well as study site [34]. MRSA colonization rate (45\%) amongst isolates from hospital personnel identified in this study was higher than the $11.4 \%$ registered for a similar group in 2013 by Rongpharpi and collaborators in Assam, Nigeria [34] [35]. The 33\% prevalence recorded for HIV-positive patients was higher than $2.4 \%$ reported in Mekelle, Northern Ethiopia [36]. Likewise the prevalence of MRSA amongst isolates from diabetes mellitus patients (39\%) was higher than 9.9\% registered by Kutlu and collaborators in 2012 [36]. These MRSA rates however fall within the $0 \%-59 \%$ range docu- 
mented in a systematic review 104 studies describing MRSA nasal carriage in several countries in Africa [37]. Discrepancy in results has been attributed to the level of compliance to control measures such as hand washing and techniques used for MRSA detection. The highest prevalence was recorded at the Yaounde Central Hospital which was the most crowded hospital facility among the study sites. Overcrowding is considered a favourable condition for the spread of MRSA through hand contact with contaminated surfaces or otherwise through the air. MRSA are resistant to several classes of antibiotics, rendering treatment of simple infections complicated and costly. Antimicrobial resistance is enhanced by ease in drug acquisition, automedication and poor hygiene.

Molecular characterisation of MRSA isolates revealed community-associated SCCmec type IV as the predominant type among the study population and study sites. A similar result was reported by Abdul et al., 2015 who identified SCCmec type IV as the leading type between SCCmec type IV and type V [38]. SCCmec type IV has been identified as the most virulent type because it carries a mecA gene, it has a functional recombinase and it is most mobile thus can be transmitted easily to MSSA within the community. Contrary to these findings, SCCmec $\mathrm{V}$ was reported as being the major type among MRSA isolated from the nares of Egyptian and Saudi Arabian outpatients. This variation could be due to the different multiplex-polymerase chain reaction (PCR) methods used in the assignment of SCCmec types as highlighted by Antiabong et al., 2016 [33]. The detection of hospital associated SCCmec type II among isolates from HIV positive and diabetes mellitus patients has been previously reported. This implies that community acquired-MRSA and hospital acquired-MRSA isolates might no longer be differentiated based only on SCCmec genotypes [29]. The circulation of MRSA strains within communities and hospitals may be driven by routine visits of patients and their family members (patients' care givers) to the hospital.

The frequency of MSSA/PVL and MRSA/PVL isolates from the three study populations were higher than reported prevalences of less than $10 \%$ in European countries, but falls within the $17 \%$ to $70 \%$ range reported for Madagascar, Morocco, Niger and Senegal [29]. While the MRSA/PLV prevalence for hospital personnel (41\%) was comparable to the rate in Algiers (44\%), rates for HIV-positive patients (35\%) and diabetes mellitus patients (24\%) were higher than has been documented in other parts of Africa [39]. Given that $l u k S$ - $P V$ is phage mediated and can easily be transmitted between isolates, this may explain high prevalence rates of gene among strains in Africa where monitoring is limited. The MRSA/PVL strains sequenced were categorized as CC8, CC121 and CC1289. Most MSSA/PVL isolates were obtained from HIV-positive patients and those sequenced were classified in clonal complexes CC8, CC15, CC72 and CC121. The CC121 clone is a known African pandemic clone common in Nigeria, Togo, South Africa and Cameroon [38]. The relatively high rates of both MSSA/PVL and MRSA/PVL strains among asymptomatic carriage isolates may serve as important endogenous reservoirs for subsequent transmission to sterile body sites and an important epidemiological focal point for possible dissemination within 
the community. This may worsen clinical outcomes in financially over burden individuals and healthcare facilities.

The predominant agr type in all three populations was agr type I, same as results by Meysam et al., 2014 who reported 43.3\% for agr type I [40]. In another study conducted by Van Leeuwen et al., 2000, 71\% of 192 S. aureus carrier strains were classified as agr I [41]. The role played by agr group I in staphylococcal infections in high risk populations need to be elucidated in future studies.

The eighteen different pulsotypes generated, grouped isolates irrespective of study population. Three isolates from hospital personnel from the Yaounde General Hospital [ $n=2]$ and the Biyem-Assi District Hospital [ $=1]$, clustered in the same pulsotype with the same band patterns at $80 \%$ homology. This indicates that intra- and inter-hospital transmission of strains, highlighting the need for improvement of hospital hygiene and hand hygiene of hospital personnel.

Twelve representative isolates were classified into six pandemic STs $(5,8,15$, 2572 and 121) based on pulsotype patterns generated by PFGE. All of these clones have been described as pandemic clones in previous studies from other African countries, with ST5 and ST15 being predominant in West and Central Africa. ST15 was reported in Mali, Gabon and Germany as the most prevalent serotype in asymptomatic carriers. The PVL-positive ST121 (MRSA-IV) clone detected, is prevalent in Africa and was also reported in Asia as a paediatric epidemic clone. [31] In addition to typical STs, three atypical STs $(508,699$, and 1289) were identified. ST1289 (SCCmecV/PLV) which is a single-locus variant of ST88, with characteristics of an epidemic clone, was detected in 2008 among clinical isolates [15]. Similarly, Schaumburg et al., 2011, found ST508 to be significantly associated with asymptomatic carriage $(p$-value $=0.024)$ amongst isolates from Gabon.

\section{Conclusion}

Hospital personnel, HIV-positive and diabetes mellitus patients in Yaounde represent an important reservoir for MRSA, MRSA-PVL and MSSA-PVL strains. The virulence factor qgr type I was common among study populations. Studying the possible role of agr I in staphylococcal infections may provide insight for controlling risk factors among high risk patients. Isolates were genetically diverse with MLST revealing pandemic ST88 as being predominant and three atypical STs $(508,699$, and 1289). The detection of uncommon STs especially ST1289 with characteristic of an epidemic clone has provided an insight into the necessity for routine monitoring to prevent spread and disease outbreaks. The clonal diversity of $S$. aureus reported in this study and the detection of some epidemic strains serve as the basis for informed decisions on better patient management.

\section{Acknowledgements}

We acknowledge assistance from the Center for the Study and Control of Com- 
municable Diseases of the Faculty of Medicine and Biomedical Sciences, The University of Yaounde 1, Cameroon and the Department of Medical Microbiology, University of Pretoria, South Africa for allowing us to conduct analyses at their facilities.

\section{Funding}

This work was partially financed by HIV-Research Trust, United Kingdom. The grant was used for molecular analysis techniques and purchase of reagents.

\section{Availability of Data and Materials}

The data used and or analysed during the current study are available from the corresponding author on reasonable request.

\section{Authors' Contributions}

AE designed the study with input from ME and ELM SKS. EM, GI, MM. CH collected samples from participants and isolated Staphylococcus aureus. AE, ELM, MCOA, MT and HGK did the molecular analysis. AE and MK did the PFGE. AE, JA and CF performed the MLST. MH and CF did the statistical analysis. AE, ME, ELM and JA wrote the first draft of the manuscript and all authors contributed to and had final approval of the article.

\section{Consent for Publication}

Not applicable.

\section{Ethics Approval}

Ethical clearance (No. 2014/07/475/CEA/CNERSH/SP) was obtained from the Cameroon National Ethics Committee for Human Health Research. Authorizations were obtained from recruitment sites and only participants who signed the assent form were enrolled in the study.

\section{Conflicts of Interest}

The authors declare that they have no competing interests.

\section{References}

[1] Murray, P., Baron, E., Jorgensen, J., Pfaller, M. and Yolken, R. (2003) Manual of Clinical Microbiology. 8th Edition, Vol. 1, American Society for Microbiology Press, Washington DC, 304-404.

[2] Boucher, H., Miller, L.G. and Razonable, R.R. (2010) Serious Infections Caused by Methicillin-Resistant Staphylococcus aureus. Clinical Infectious Diseases, 51, S183-S197. https://academic.oup.com/cid/article/51/Supplement 2/S183/382346 https://doi.org/10.1086/653519

[3] van den Akker, E.L.T., et al. (2006) Staphylococcus aureus Nasal Carriage Is Associated with Glucocorticoid Receptor Gene Polymorphisms. The Journal of Infectious Diseases, 194, 814-818. 
https://academic.oup.com/jid/article/194/6/814/865388 https://doi.org/10.1086/506367

[4] Emonts, M., et al. (2008) Host Polymorphisms in Interleukin 4, Complement Factor $\mathrm{H}$, and C-Reactive Protein Associated with Nasal Carriage of Staphylococcus aureus and Occurrence of Boils. The Journal of Infectious Diseases, 197, 1244-1253. https://academic.oup.com/jid/article/197/9/1244/869861 https://doi.org/10.1086/533501

[5] Mulcahy, M.E., Geoghegan, J.A., Monk, I.R., O’Keeffe, K.M., Walsh, E.J., Foster, T.J., et al. (2012) Nasal Colonisation by Staphylococcus aureus Depends upon Clumping Factor B Binding to the Squamous Epithelial Cell Envelope Protein Loricrin. PLOS Pathogens, 8, e1003092.

https://journals.plos.org/plospathogens/article?id=10.1371/journal.ppat.1003092

[6] Wertheim, H.F., Vos, M.C., Ott, A., Belkum, A., Voss, A., Kluytmans, J.A., et al. (2004) Risk and Outcome of Nosocomial Staphylococcus aureus Bacteraemia in Nasal Carriers versus Non-Carriers. The Lancet, 364, 703-705.

https://doi.org/10.1016/S0140-6736(04)16897-9

[7] Holtfreter, S., Radcliff, F.J., Grumann, D., Read, H., Johnson, S., Monecke, S., et al. (2013) Characterization of a Mouse-Adapted Staphylococcus aureus Strain. PLoS $O N E$, 8, e71142. https://doi.org/10.1371/journal.pone.0071142

[8] Kiser, K.B., Cantey-Kiser, J.M. and Lee, J.C. (1999) Development and Characterization of a Staphylococcus aureus Nasal Colonization Model in Mice. Infection and Immunity, 67, 5001-5006.

[9] Foster, T.J. (2004) The Staphylococcus aureus "Superbug". Journal of Clinical Investigation, 114, 1689-1689. https://www.jci.org/articles/view/23825

[10] Foster, T.J. (2005) Immune Evasion by Staphylococci. Nature Reviews Microbiolo$g y, 3,948-958$. https://www.nature.com/articles/nrmicro1289

[11] Le Loir, Y., Baron, F. and Gautier, M. (2003) Staphylococcus aureus and Food Poisoning. Genetics and Molecular Research, 2, 63-76.

[12] Zanger, P., Nurjadi, D., Vath, B. and Kremsner, P.G. (2011) Persistent Nasal Carriage of Staphylococcus aureus Is Associated with Deficient Induction of Human $\beta$-Defensin 3 after Sterile Wounding of Healthy Skin in Vivo. Infection and Immunity, 79, 2658-2662.

[13] Popoola, V.O., Carroll, K.C., Ross, T., Reich, N.G., Perl, T.M. and Milstone, A.M. (2013) Impact of Colonization Pressure and Strain Type on Methicillin-Resistant Staphylococcus aureus Transmission in Children. Clinical Infectious Diseases, 57, 1458-1460. https://doi.org/10.1093/cid/cit542

[14] Lambert, M.-L., Suetens, C., Savey, A., Palomar, M., Hiesmayr, M., Morales, I., et al. (2011) Clinical Outcomes of Health-Care-Associated Infections and Antimicrobial Resistance in Patients Admitted to European Intensive-Care Units: A Cohort Study. The Lancet Infectious Diseases, 11, 30-38. https://doi.org/10.1016/S1473-3099(10)70258-9

[15] Villacian, J.S., Barkham, T., Earnest, A. and Paton, N.I. (2004) Prevalence of and Risk Factors for Nasal Colonization with Staphylococcus aureus among Human Immunodeficiency Virus-Positive Outpatients in Singapore. Infection Control \& Hospital Epidemiology, 25, 438-440. https://doi.org/10.1086/502420

[16] Mathews, W.C., Caperna, J.C., Barber, R.E., Torriani, F.J., Miller, L.G., May, S., et al. (2005) Incidence of and Risk Factors for Clinically Significant Methicillin-Resistant Staphylococcus aureus Infection in a Cohort of HIV-Infected Adults. Journal of 
Acquired Immune Deficiency Syndromes, 40, 155-160. https://doi.org/10.1097/01.qai.0000179464.40948.b9

[17] Udo, E.E. and Grubb, W.B. (1993) Genetic Analysis of Methicillin-Resistant Staphylococcus aureus from a Nigerian Hospital. Journal of Medical Microbiology, 38, 203-208.

https://www.microbiologyresearch.org/content/journal/jmm/10.1099/00222615-38$\underline{3-203}$

[18] Shibabaw, A., Abebe, T. and Mihret, A. (2013) Nasal Carriage Rate of Methicillin Resistant Staphylococcus aureus among Dessie Referral Hospital Health Care Workers; Dessie, Northeast Ethiopia. Antimicrobial Resistance \& Infection Control, 2, Article No. 25. https://aricjournal.biomedcentral.com/articles/10.1186/2047-2994-2-25

[19] Eyoh, A.B., Eyoh, A.B., Toukam, M., Gonsu, H., Fokunang, C., Lyonga, E.E., et al. (2013) Antibiotics Susceptibility Profile of Staphylococcus aureus Isolated from the Anterior Nares of Hospital Personnel in Yaounde, Cameroon. Health Sciences and Disease, 14, 1-5. https://www.hsd-fmsb.org/index.php/hsd/article/view/146

[20] Matussek, A., Taipalensuu, J., Einemo, I.-M., Tiefenthal, M. and Löfgren, S. (2007) Transmission of Staphylococcus aureus from Maternity Unit Staff Members to Newborns Disclosed through Spa Typing. American Journal of Infection Control, 35, 122-125. https://doi.org/10.1016/j.ajic.2006.08.009

[21] Valiquette, L., Chakra, C.N.A. and Laupland, K.B. (2014) Financial Impact of Health Care-Associated Infections: When Money Talks. Canadian Journal of Infectious Diseases and Medical Microbiology, 25, Article ID: 279794. https://www.hindawi.com/journals/cjidmm/2014/279794

[22] Revelas, A. (2012) Healthcare-Associated Infections: A Public Health Problem. Journal of the Nigeria Medical Association, 53, 59-64. https://doi.org/10.4103/0300-1652.103543

[23] Kolawole, D.O., et al. (2013) Characterization of Colonizing Staphylococcus aureus Isolated from Surgical Wards' Patients in a Nigerian University Hospital. PLoS ONE, 8, e68721.

https://journals.plos.org/plosone/article?id=10.1371/journal.pone.0068721

[24] David, M.Z. and Daum, R.S. (2010) Community-Associated Methicillin-Resistant Staphylococcus aureus. Epidemiology and Clinical Consequences of an Emerging Epidemic. Clinical Microbiology Reviews, 23, 616-687.

[25] Shepheard, M.A., et al. (2013) Historical Zoonoses and Other Changes in Host Tropism of Staphylococcus aureus, Identified by Phylogenetic Analysis of a Population Dataset. PLOS ONE, 8, e62369.

https://journals.plos.org/plosone/article?id=10.1371/journal.pone.0062369

[26] Schaumburg, F., Alabi, A.S., Peters, G. and Becker, K. (2014) New Epidemiology of Staphylococcus aureus Infection in Africa. Clinical Microbiology and Infection, 20, 589-596.

https://www.clinicalmicrobiologyandinfection.com/article/S1198-743X(14)61144-7/ $\underline{\text { fulltext }}$

[27] AlFouzan, W., Al-Haddad, A., Udo, E., Mathew, B. and Dhar, R. (2013) Frequency and Clinical Association of Panton-Valentine Leukocidin-Positive Staphylococcus aureus Isolates: A Study from Kuwait. Medical Principles and Practice, 22, 245-249. https://www.karger.com/Article/FullText/343906 https://doi.org/10.1159/000343906

[28] Ellington, M.J., Ganner, M., Smith, I.M., Perry, C., Cookson, B.D. and Kearns, A.M. 
(2010) Panton-Valentine Leucocidin-Related Disease in England and Wales. Clinical Microbiology and Infection, 16, 86-88. https://doi.org/10.1111/j.1469-0691.2009.02887.x

[29] Enright, M.C., Day, N.P., Davies, C.E., Peacock, S.J. and Spratt, B.G. (2000) Multilocus Sequence Typing for Characterization of Methicillin-Resistant and Methicillin-Susceptible Clones of Staphylococcus aureus. Journal of Clinical Microbiology, 38, 1008-1015. https://pubmed.ncbi.nlm.nih.gov/10698988

[30] Al-Talib, H., et al. (2009) A Pentaplex PCR Assay for the Rapid Detection of Methicillin-Resistant Staphylococcus aureus and Panton-Valentine Leucocidin. BMC Microbiology, 9, Article No. 113.

https://bmcmicrobiol.biomedcentral.com/articles/10.1186/1471-2180-9-113

[31] Zhang, K.Y., McClure, J.-A., Elsayed, S., Louie, T. and Conly, J.M. (2008) Novel Multiplex PCR Assay for Simultaneous Identification of Community-Associated Methicillin-Resistant Staphylococcus aureus Strains USA300 and USA400 and Detection of mecA and Panton-Valentine Leukocidin Genes, with Discrimination of from Coagulase-Negative Staphylococci. Journal of Clinical Microbiology, 46, 1118-1122. https://pesquisa.bvsalud.org/portal/resource/e/mdl-18160447

[32] Kondo, Y., et al. (2007) Combination of Multiplex PCRs for Staphylococcal Cassette Chromosome mec Type Assignment: Rapid Identification System for mec, ccr, and Major Differences in Junkyard Regions. Antimicrobial Agents and Chemotherapy, 51, 264-274.

[33] Ruppé, E., et al. (2009) Diversity of Staphylococcal Cassette Chromosome mec Structures in Methicillin-Resistant Staphylococcus epidermidis and Staphylococcus haemolyticus Strains among Outpatients from Four Countries. Antimicrobial Agents and Chemotherapy, 53, 442-449.

[34] Bhutia, K.O., Singh, T.S., Biswas, S. and Adhikari, L. (2012) Evaluation of Phenotypic with Genotypic Methods for Species Identification and Detection of Methicillin Resistant in Staphylococcus aureus. International Journal of Applied and Basic Medical Research, 2, 84-91. https://doi.org/10.4103/2229-516X.106348

[35] Rongpharpi, S.R., Hazarika, N.K. and Kalita, H. (2013) The Prevalence of Nasal Carriage of Staphylococcus aureus among Healthcare Workers at a Tertiary Care Hospital in Assam with Special Reference to MRSA. Journal of Clinical and Diagnostic Research, 7, 257-260. https://doi.org/10.7860/JCDR/2013/4320.2741

[36] Kutlu, S.S., et al. (2012) Prevalence and Risk Factors for Methicillin-Resistant Staphylococcus aureus Colonization in a Diabetic Outpatient Population: A Prospective Cohort Study. American Journal of Infection Control, 40, 365-368.

https://www.ajicjournal.org/article/S0196-6553(11)00831-5/fulltext

[37] Abdulgade, S.M., Shittu, A.O., Nicol, M.P. and Kaba, M. (2015) Molecular Epidemiology of Methicillin-Resistant Staphylococcus aureus in Africa: A Systematic Review. Frontiers in Microbiology, 6, 348. https://www.frontiersin.org/articles/10.3389/fmicb.2015.00348/full

[38] Hannan, A., Javed, F., Saleem, S., Tahira, K. and Jahan, S. (2015) Frequency of Staphylococcal Cassette Chromosome mec Type IV and Type V in Clinical Isolates of Methicillin Resistant Staphylococcus aureus. Open Journal of Medical Microbiology, 5, 69-75. https://doi.org/10.4236/ojmm.2015.52008

[39] Breurec, S., et al. (2011) Epidemiology of Methicillin-Resistant Staphylococcus aureus Lineages in Five Major African Towns: Emergence and Spread of Atypical Clones. Clinical Microbiology and Infection, 17, 160-165.

https://www.clinicalmicrobiologyandinfection.com/article/S1198-743X(14)61655-4/ 
fulltext

[40] Bibalan, M.H., Shakeri, F., Javid, N., Ghaemi, A. and Ghaemi, E.A. (2014) Accessory Gene Regulator Types of Staphylococcus aureus Isolated in Gorgan, North of Iran. Journal of Clinical and Diagnostic Research, 8, DC07-DC09.

[41] van Leeuwen, W., van Nieuwenhuizen, W., Gijzen, C., Verbrugh, H. and van Belkum, A. (2000) Population Studies of Methicillin-Resistant and -Sensitive Staphylococcus aureus Strains Reveal a Lack of Variability in the agrD Gene, Encoding a Staphylococcal Autoinducer Peptide. Journal of Bacteriology, 182, 5721-5729.

https://doi.org/10.1128/JB.182.20.5721-5729.2000 